\title{
Adhesion of A. actinomycetemcomitans to Host Components of the Extracellular Matrix
}

\author{
Fereshteh Azari ${ }^{1}$, Michael Radermacher ${ }^{1}$, Keith Mintz ${ }^{2}$ and Teresa Ruiz ${ }^{1}$ \\ 1. Dept. of Molecular Physiology and Biophysics, University of Vermont, Burlington, VT 05405 \\ 2. Dept. of Microbiology and Molecular Genetics, University of Vermont, Burlington, VT 05405
}

The periodontal pocket is colonized by bacteria that exist in the dental biofilm or plaque. Apical migration of the pocket epithelium deepens the periodontal pocket. This first step is followed by the resorption of the alveolar bone, which leads initially e to tooth mobility and subsequently to tooth loss. Aggregatibacter actinomycetemcomitans, a gram-negative bacterium, localized in the periodontal pocket, is involved in causing periodontitis and other serious systemic diseases, e.g. soft abscesses, pneumonia, urinary tract infection, infective endocarditis, and the formation of atherosclerotic plaque [1]. Adhesion to host tissue, such as extracellular matrix, is considered an essential event in the pathogenic process of the infectious disease of this bacterium. SEM examination of trypsin treated rabbit mitral valves showed large numbers of $A$. actinomycetemcomitans (Aa) bound to the exposed collagen fibers [2]. Adhesion to collagen is mainly mediated by EmaA (Extracellular matrix protein adhesin A), which is a non fimbrial adhesin that forms antenna-like appendages on the surface of the bacteria. These appendages are absent on the surface of the emaA mutant strain, which also lacks the ability to bind collagen [3]. Complementation of the mutant strain with a plasmid containing the emaA gene restores both the surface appendages and the collagen binding activity.

EmaA has a molecular mass of $202 \mathrm{kDa}$ and belongs to the family of trimeric autotransporter adhesins. Members of this family possess a characteristic amino-terminal signal peptide sequence; highly conserved sequences that serve as transitions between secondary structural elements (neck sequence); a stalk domain with a high probability of coiled-coil formation, followed by a membrane anchor domain. Deletion mapping combined with 3D structure determination by electron tomography of EmaA have shown that the distal $30 \mathrm{~nm}$ region of the structure is composed of amino acids 57-760 from three monomers (the signal peptide comprises residues 1-56). This region is further divided into three subdomains, SI, SII and SIII and exhibits the collagen binding activity of the adhesin [4,5]. The bacteria carrying a deletion in amino acids 70-386 of the EmaA sequence showed a marked decrease in collagen binding activity and lack subdomains SI and SII. Our goal is to determine the molecular mechanisms involved in the interaction of EmaA with collagen to provide a better understanding of the first step in the infection process.

A large number of experimental conditions were investigated to form collagen fibrils from acid soluble rat type I collagen. Optimum size of the fibrils was obtained from a $0.1 \mathrm{mg} / \mathrm{ml}$ collagen suspension in 10 $\mathrm{mM}$ sodium phosphate and $150 \mathrm{mM} \mathrm{NaCl}$ buffer with $\mathrm{pH} 7.4$ after $30-45$ min incubation at $22^{\circ} \mathrm{C}$. Visualization of $\mathrm{Aa} /$ collagen mixtures by light and electron microscopy revealed interactions between the bacteria and the collagen fibrils (Fig.1A-B). We probed this interaction in more detail by performing electron tomography. Single axis tomography tilt series were acquired from whole-mount negatively stained preparations of the Aa/collagen samples. Each series was recorded over a $\pm 70^{\circ}$ angular range in $2^{\circ}$ increments with $0.3 \mathrm{~nm}$ pixel size. Local patch tracking alignment of the projections and tomographic reconstructions were carried out using IMOD [6]. In the reconstructed tomograms, EmaA was clearly recognizable protruding from the bacterial surface. In addition, collagen fibrils of various size and 
lengths were observed in the close vicinity of EmaA (Fig.2A, bottom-left region of the black box). Subvolumes containing EmaA and collagen were extracted from tomograms. The analysis of the Z-slices from subvolumes clearly shows that EmaA is in close contact with the collagen fibrils (Fig.2B). Investigation of more than 100 different tomograms indicates that EmaA binds to collagen at different position, mostly within the functional domain (the $30 \mathrm{~nm}$ distal end of the appendage). In order to determine which EmaA subdomains (SI, SII or SIII) interact with collagen, we need to segment EmaA and collagen from the extracted subvolumes and compare them to our 3D structure of EmaA [5]. In the future, we will also characterize the interaction of EmaA with collagen at the molecular level. The information obtained will bring us closer to understanding this interaction and to identify novel methods to reduce the pathogenic potential of this bacterium.

References:

[1] J Zambon, J Clin Periodontol 12(1985), p.1.

[2] G Tang et al., Infect Immun 76 (2008), p. 2316.

[3] T Ruiz et al. Infect Immun 74 (2006), p. 6163.

[4] F Azari et al, J struct Biol 177 (2012), p. 439.

[5] C Yu et al, J Bacteriol 191(2009), p. 6253.

[6] J Kremer et al, J struct Biol 116 (1996), p. 71.

[7] This work was supported by NIH grant R01 DE017474 (T.R.).
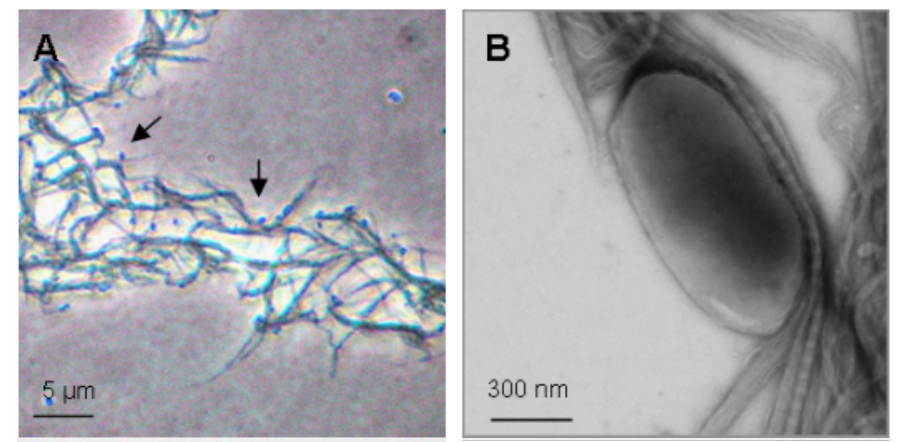

Figure 1. Visualization of Aa/collagen samples by (A) light microscopy and (B) electron microscopy. Both figures shows bacteria bound to collagen fibrils. Arrows point to bacteria in (A).
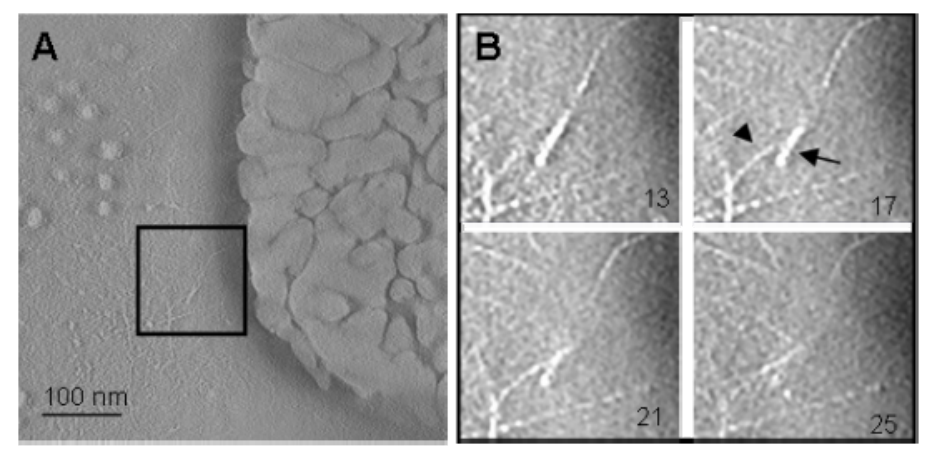

Figure 2. Interaction of EmaA with collagen fibrils. (A) 3D tomogram of a bacterium/collagen sample. (B) Z-slices (slices:13,17,21,25) extracted from a tomogram subvolume containing EmaA interacting with collagen (black box in (A)). The arrow points to EmaA and the arrow head to collagen. Each slice represents $0.308 \mathrm{~nm}$ in the sample scale. 